\title{
Estudo de Adsorção para Remoção do Corante Remazol Preto B em Solução Aquosa Utilizando Carvão Ativado
}

\author{
lara A. Freire, Gabriel N. C. Nova \& Nelson M. L. Filho
}

\begin{abstract}
É de grande importância ambiental que efluentes de indústrias têxteis sejam tratados antes de serem descartados, visto que estes apresentam corantes que são agentes poluidores quando em contato com a natureza. Um processo bastante utilizado para retirar estas substancia do meio é a adsorção, pela simplicidade de operação, por ser economicamente viável em proporções industriais permitindo a utilização de materiais de baixo custo e alta capacidade de remoção de solutos. Este trabalho teve como objetivo avaliar a remoção do corante Remazol preto B através deste processo utilizando carvão ativado como adsorvente, a fim DE determinar isotermas de equilíbrio do processo, as curvas cinéticas de adsorção e avaliar a capacidade de remoção de corantes em um efluente sintético. Os experimentos foram conduzidos em um reator de vidro em leito de lama num processo em batelada a velocidade constante de agitação de $300 \mathrm{rpm}$ à $32^{\circ} \mathrm{C}$ onde foram colocados o carvão e a solução sintética do corante. Amostras foram retiradas com um filtro poroso e realizada leitura em um espectrofotômetro num comprimento de onda de $595 \mathrm{~nm}$. No experimento de cinética, identificado como sendo de $1^{\text {a }}$ ordem, o equilíbrio foi atingido a partir de $240 \mathrm{~min}$ com remoção de $84 \%$, estabilizando-se em 330 min removendo $90 \%$ do corante. O modelo de isoterma de Langmuir foi o que melhor se ajustou ao comportamento da adsorção do RB na superfície do adsorvente e a capacidade máxima adsortiva obtida foi de 5,656 mg.g-1 para o corante sobre o carvão.
\end{abstract}

\section{Palavras-chave: Adsorção; Remazol; Efluente Têxtil; Carvão Ativado.}

It is of great environmental importance that effluents from textile industries are treated before being discarded, since they present dyes that are polluting agents when in contact with nature. A process widely used to remove these substances from the medium is the adsorption, for the simplicity of operation, to be economically viable in industrial proportions allowing the use of materials of low cost and high capacity of removal of solutes. The objective of this work was to evaluate the removal of Remazol dye B through this process using activated charcoal as an adsorbent in order to determine process equilibrium isotherms, adsorption kinetic curves and to evaluate the dye removal capacity in a synthetic effluent. The experiments were conducted in a slurry bed reactor in a batch process at constant stirring speed of $300 \mathrm{rpm}$ at $32^{\circ} \mathrm{C}$ where the charcoal and the synthetic dye solution were placed. Samples were drawn with a porous filter and read on a spectrophotometer at a wavelength of $595 \mathrm{~nm}$. In the kinetic experiment, identified as being of the first order, the equilibrium was reached from 240 min with $84 \%$ removal, stabilizing in 330 min removing $90 \%$ of the dye. The Langmuir isotherm model was the best fit for the adsorption behavior of RB on the adsorbent surface and the maximum adsorptive capacity obtained was $5.656 \mathrm{mg}$.g- 1 for the dye on the charcoal.

Keywords: Adsorption; Remazol; Textile Effluent; Activated Charcoal. 


\section{Introdução}

A indústria utiliza a água para diversos processos, tais como lavagens das máquinas, pisos e tubulações, refrigeração e geradores de vapor, incorporação direta nos produtos ou nas etapas da produção, esgotos sanitários, entre outros. No caso da indústria têxtil a água é um dos elementos básicos no processo de tingimento das peças, no qual a qualidade da água é alterada devido à adição de substâncias químicas (corantes). Uma pequena parte destes não se fixa às fibras dos tecidos continuando por isso, dissolvidos na água e esta, quando é lançada sem o devido tratamento para o ambiente constitui um agente poluidor.

Os corantes podem ser visíveis em alguns casos em concentrações tão baixas quanto um ppm (GUARATINI, 2000) e em pequenas quantidades afetam a transparência da água e a solubilidade dos gases. Esses pigmentos possuem, em geral, alta estabilidade biológica, alto peso molecular e presença de anéis aromáticos. Devido à alta toxicidade seu tratamento é necessário, os métodos mais utilizados para remoção de corantes de efluentes são processos biológicos e físico-químicos como precipitação, coagulação, adsorção, etc (GONÇALVES, 2004).

O corante utilizado neste trabalho foi o Remazol Preto B 133\% GRAN (OP.PN10503, $\mathrm{C}_{26} \mathrm{H}_{21} \mathrm{~N}_{5} \mathrm{O}_{19} \mathrm{~S}_{6} \mathrm{Na}_{4}, 991,82$ g.mol ${ }^{-1}$, Figura 1), composto da classe do azo corantes (apresentam um ou mais grupamentos $-\mathrm{N}=\mathrm{N}$ - ligados a sistemas aromáticos), muito utilizado na indústria têxtil, sendo cerca de $60 \%$ dos corantes, utilizados na área, deste tipo (KUNZ, 2002). Este corante apresenta 2 grupos sulfonato e outros dois grupo sulfatoetilsulfona, com cargas negativas, mesmo em soluções altamente ácidas, devido ao seu pKa com valores menores que zero (Lima et al, 2008).

$\mathrm{O}$ método de tratamento escolhido para a remoção deste corante foi a adsorção. Segundo Soto et al (2011), os processos adsortivos se destacam por sua simplicidade de projeto e operação, insensibilidade a substâncias tóxicas, facilidade de regeneração e baixo custo, além de evitar a utilização de solventes tóxicos.

O objetivo desta pesquisa foi avaliar a remoção do corante Remazol preto B através do processo de adsorção

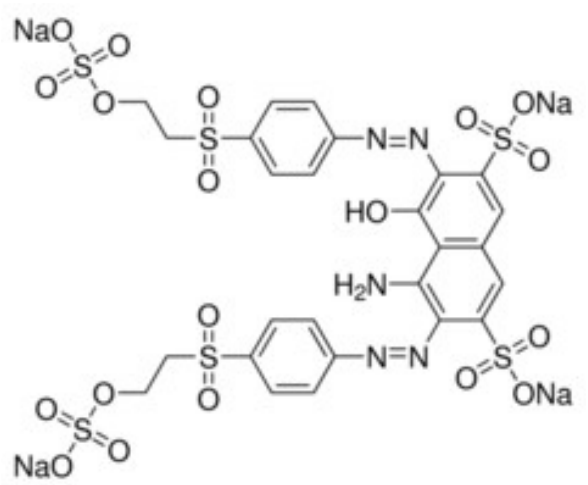

Figura 1. Fómula estrutural do corante têxtil Remazol Preto B (RB).

utilizando carvão ativado, a fim de determinar isotermas de equilíbrio do processo, as curvas cinéticas de adsorção e avaliar a capacidade de remoção de corantes em um efluente sintético.

\section{Metodologia}

Neste trabalho utilizou-se, como técnica de separação do corante, o método de adsorção, para tal foi utilizado carvão ativado como adsorvente. A fim de determinar sua capacidade adsortiva em meio à solução corante, as propriedades físico-quimicas deste adsorvente foram caracterizadas partir das seguintes técnicas: análise termogravimétrica (TGA), ponto de carga zero $\left(\mathrm{pH}_{\mathrm{PCZ}}\right)$ e método de caracterização textural (Brunauer, Emmett, Teller - BET).

\section{PROCEDIMENTO EXPERIMENTAL}

A concentração inicial da solução sintética foi preparada a partir do corante comercial em pó Remazol Preto B (RB) dissolvido em água destilada numa concentração de $400 \mathrm{mg} \cdot \mathrm{L}^{-1}$. As soluções de Remazol utilizadas posteriormente no estudo foram obtidas através da diluição desta solução inicial.

Todos os experimentos de cinética de adsorção foram conduzidos em um reator de vidro em leito de lama num 
processo em batelada, a uma velocidade constante de agitação de $300 \mathrm{rpm}$, num banho termostático a $32^{\circ} \mathrm{C}$ onde foram colocados a solução sintética de corante e o adsorvente. Foram realizadas retiradas de amostras com filtro poroso, aproximadamente $5 \mathrm{~mL}$ cada, na primeira hora foram retiradas 14 amostras, na segunda hora, 4 (a cada 15 minutos) e nas próximas horas a cada 30 minutos até a estabilização da reação. Onde foram realizadas leituras de concentração num espectrofotômetro, Cary 50 Bio UV/ visível, no comprimento de onda de $595 \mathrm{~nm}$. A quantidade de massa de corante adsorvida por massa de carvão no equilíbrio foi calculada utilizando a Equação 1:

$$
q=\frac{\left(C_{0}-C\right)}{m} . V
$$

Sendo q a quantidade do adsorbato em $\mathrm{mg}$ de adsorvato. $\mathrm{g}^{-1}$ de adsorvente, $\mathrm{C}_{0}$ a concentração inicial (mg. $\left.\mathrm{L}^{-1}\right), \mathrm{C}$ a concentração no momento da retirada da amostra (mg. $\left.\mathrm{L}^{-1}\right), \mathrm{V}$ o volume da solução (L) e m a massa de adsorvente $(\mathrm{g})$.

O modelo de adsorção utilizado para avaliar os dados de equilíbrio do processo foi o de Langmuir-Freundlich (Equação 2). Para este estudo foram realizados experimentos utilizando $2,8 \mathrm{~g}$ de carvão ativado em contato com $700 \mathrm{ml} \mathrm{de}$ solução sintética do corante nas concentrações de $4 ; 16 ; 28$ e $40 \mathrm{mg} \cdot \mathrm{L}^{-1}$.

$$
q_{e}=\frac{q_{\max } K_{L F} C_{e}^{1 / n}}{1+K_{L F} C_{e}^{1 / n}}
$$

Onde qe é a quantidade de adsorbato adsorvido no equilíbrio (mg. $\left.\mathrm{L}^{-1}\right)$, qmax a capacidade máxima adsortiva, KLF é a constante de Langmuir-Freundlich, $\mathrm{Ce}$ a concentração no equilíbrio (mg. $\left.\mathrm{L}^{-1}\right)$ e $\mathrm{n}$ a ordem da equação.

\section{CARACTERIZAÇÃO DO ADSORVENTE}

Análise Termogravimétrica: $O$ experimento foi realizado seguindo o procedimento adotado pelo Laboratório de Processos Catalíticos (LPC/UFPE). A amostra foi submetida a ensaios termogravimétricos para obtenção dos intervalos e percentuais de perdas de massa através do calor. A análise foi realizada no LATECLIM/UFPE, em uma termobalança Perkin Elmer, modelo STA 6000, com taxa de aquecimento de $20^{\circ}$ C.min-1, sob fluxo de $20 \mathrm{~mL} \cdot \mathrm{min}^{-1}$ de N2. A massa de material utilizado nos cadinhos de platina foi fixada em $10 \mathrm{mg}$ e as mesmas foram aquecidas de $30^{\circ} \mathrm{C}$ a $800^{\circ} \mathrm{C}$. Os resultados obtidos foram tratados com o auxílio do software Pyris Data Analysis, versão 11.

Ponto de carga zero: Foi determinado colocando-se $0,1 \mathrm{~g}$ do material em contato com água destilada, numa faixa de $\mathrm{pH}$ entre 2 e 11 , que foram ajustados com soluções de ácido clorídrico $\left(0,1 \mathrm{~mol} . \mathrm{L}^{-1}\right)$ e hidróxido de sódio $\left(0,1 \mathrm{~mol} . \mathrm{L}^{-1}\right)$ com auxílio de um pHmetro, sob agitação de $300 \mathrm{rpm}$. O pH foi medido novamente após o tempo de contato que foi de $24 \mathrm{~h}$. Para a determinação do $\mathrm{pH}_{\mathrm{PCZ}}$, foi construído o gráfico ( $\mathrm{pHfinal-pHinicial)}$ vs. pHinicial, no qual a curva que intercepta o eixo do do pHincial corresponde ao ponto onde a carga superficial do material é nula (REGALBUTO, 2016).

Caracterização Textural: A área superficial específica dos materiais usados neste trabalho foi determinada através da adsorção de N2 a $77 \pm 5 \mathrm{~K}$ em um equipamento BELSORP-MINI da Bel Japan Inc, no CETENE. Para remoção da umidade da superfície da amostra, foi realizado um pré-tratamento a $333 \mathrm{~K}$ sob vácuo por 3 horas.

\section{Resultados e Discussão}

Análise Termogravimétrica: Os resultados da análise termogravimétrica do carvão ativado estão apresentados na Figura 2, onde se pode observar que o adsorvente não sofre percas significativas de massa na temperatura utilizada durante o processo descrito neste trabalho. 


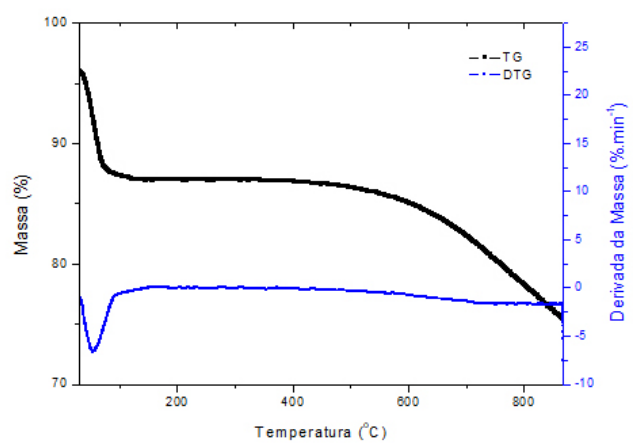

Figura 2. Análise termogravimétrica do carvão ativado.

Ponto de carga zero: Observa-se na Figura 3, que a interseção com o eixo horizontal foi no $\mathrm{pH}=7,2$, que corresponde ao $\mathrm{pH}$ no ponto de carga zero $\left(\mathrm{pH}_{\mathrm{PCZ}}\right)$. Isso significa que em $\mathrm{pH}$ acima deste valor, as cargas na superfície do carvão são negativas, favorecendo a adsorção de cátions, enquanto que para valores de $\mathrm{pH}$ abaixo de 7,2, as cargas na superficiais são positivas, favorecendo a adsorção de ânions, como é o caso do corante em estudo.

Tendo em vista este valor de $\mathrm{pH}$ no ponto de carga zero do carvão utilizado, foi escolhido trabalhar no $\mathrm{pH}$ natural da solução, visto que este tem valor menor que 7,2, viabilizando assim a adsorção do corante sobre o adsorvente.

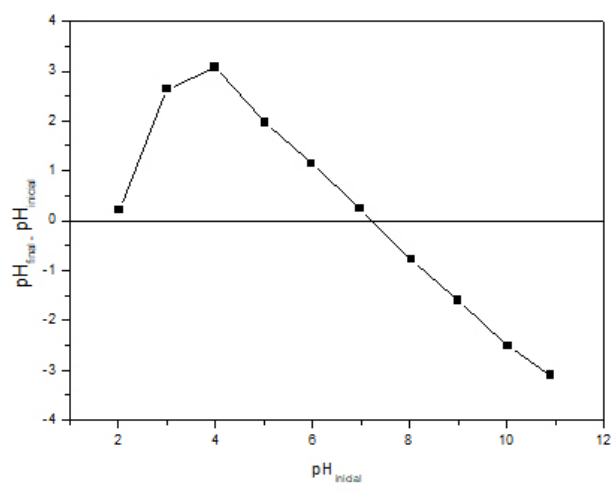

Figura 3. Ponto de carga zero do carvão ativado
Caracterização Textural: O Resultado do BET através da adsorção de $\mathrm{N}_{2}$, para o carvão ativado estão presentes na Tabela 1:

Tabela 1. Resultados obtidos por adsorção de N2.

\begin{tabular}{|c|c|c|c|}
\hline Material & $\begin{array}{c}\text { Área } \\
\text { superficial } \\
\left(\mathrm{m}^{2} \mathrm{~g}^{-1}\right)\end{array}$ & $\begin{array}{c}\text { Volume total } \\
\text { dos poros } \\
\left(\mathrm{cm}^{3} \mathrm{~g}^{-1}\right)\end{array}$ & $\begin{array}{c}\text { Diâmetro dos } \\
\text { poros }(\AA)\end{array}$ \\
\hline $\begin{array}{c}\text { Carvão } \\
\text { ativado }\end{array}$ & 829,4 & 0,476 & 11,25 \\
\hline
\end{tabular}

De acordo como a IUPAC (1982) os carvões utilizados são classificados como mesoporosos. Apresenta uma grande área superficial o que pode indicar que possui potencial para ser um bom adsorvente.

Cinética da reação: A cinética no processo de remoção do corante em contato com o carvão foi avaliada experimentalmente em ensaios entre 0 e 660 minutos. $\mathrm{O}$ tempo de equilíbrio para a adsorção se deu a partir de 240 min, onde a taxa de remoção é de aproximadamente $84 \%$, estabilizando-se a partir de $330 \mathrm{~min}$, com uma taxa de $90 \%$ de remoção do corante (Figura 4). Não foi estabelecido um tempo máximo para realização dos experimentos de equilíbrio, a fim de garantir que o mesmo tivesse sido atingido, amostras eram retiradas a cada 30 min e lidas no espectrofotômetro e só após a estabilização da reação o experimento era finalizado.

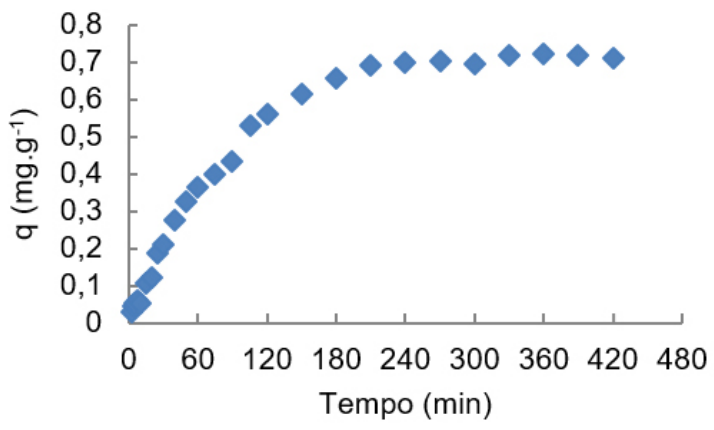

Figura 4. Comportamento cinético da adsorção do corante Remazol Preto B. $\mathrm{T}=32^{\circ} \mathrm{C}$; Velocidade de agitação $=300 \mathrm{rpm}$, massa de adsorvente: $2,8 \mathrm{~g}$; Concentração inicial da solução sintética do corante $=4 \mathrm{mg} \cdot \mathrm{L}^{-1}$. 
Equilíbrio de adsorção: $O$ modelo de LangmuirFreundlich (Equação 2) foi utilizado para representar o comportamento de adsorção do Remazol Preto B na superfície do carvão ativado. A isoterma da adsorção está representada na Figura 5 e a linearização do modelo é representada pela Figura 6, onde se pode estimar a capacidade máxima de adsorção qmax, e a constante de equilíbrio de Langmuir, Keq, representados na Tabela 2.

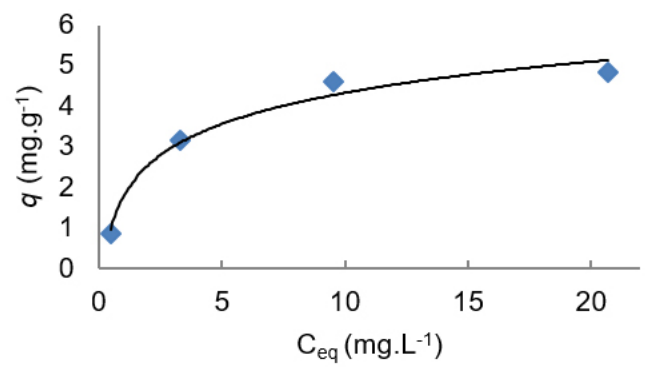

Figura 5. Isoterma de adsorção do corante Remazol Preto B sobre carvão ativado. $\mathrm{T}=32^{\circ} \mathrm{C}$; Velocidade de rotação $=300 \mathrm{rpm}$; massa do adsorvente $=2,8 \mathrm{~g}$.

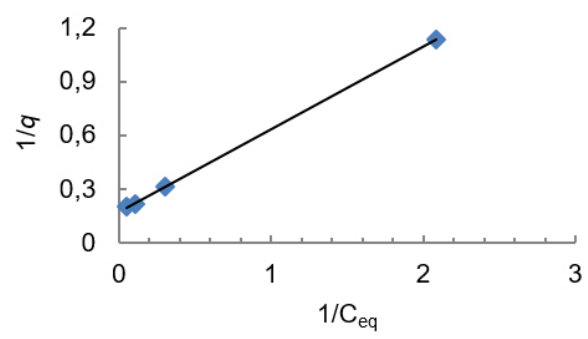

Figura 6. Linearização do modelo de Langmuir.

Observando os gráficos nota-se que o modelo de Langmuir se ajustou bem aos pontos experimentais, tendo em vista que foi obtido um bom coeficiente de correlação na linearização $\left(R_{2}=0,9998\right)$.
Tabela 2. Dados utilizados para desenvolvimento do modelo cinético de adsorção.

\begin{tabular}{|c|c|}
\hline Parâmetros & Valores \\
\hline $\mathrm{m}$ & $2,8 \mathrm{~g}$ \\
\hline $\mathrm{V}$ & $0,7 \mathrm{~L}$ \\
\hline Co & $4-40 \mathrm{mg} \cdot \mathrm{L}^{-1}$ \\
\hline qmax & $5,656 \mathrm{mg} \cdot \mathrm{g}^{-1}$ \\
\hline Keq & $0,3894 \mathrm{~L} \cdot \mathrm{mg}^{-1}$ \\
\hline
\end{tabular}

\section{Conclusões}

O estudo da remoção de corantes em soluções sintéticas demonstrou uma capacidade máxima de adsorção avaliada pela equação de Langmuir de $5,656 \mathrm{mg} \cdot \mathrm{g}^{-1}$ para o corante Remazol Preto B sobre o carvão ativado.

A avaliação da isoterma de equilíbrio conduz resultados experimentais satisfatoriamente modelado pela equação de Langmuir, sendo a constante de equilíbrio de adsorção igual a $0,3894 \mathrm{~L} \cdot \mathrm{mg}^{-1}$, avaliada nessas condições. A cinética de adsorção foi identificada como sendo de $1^{\mathrm{a}}$ ordem.

O carvão ativado apresentou uma boa capacidade adsortiva. A carga superficial positiva observada neste adsorvente, através do estudo do ponto de carga zero, demonstra a viabilidade em utiliza-lo sem variação do $\mathrm{pH}$ do meio, visto que o corante em estudo é uma substância aniônica em solução.

\section{Agradecimentos}

Aos amigos e familiares pelo apoio, incentivo e confiança. Ao professor orientador Nelson Filho pela paciência e dedicação. Ao Laboratório de Processos Catalíticos da UFPE por disponibilizar todos os materiais e equipamentos que foram necessários para a realização dessa pesquisa. A toda equipe do LPC, em especial aos amigos Bruno Santos e Thiago Emanoel. 


\section{Referências Bibliográficas}

1. Gonçalves, I.; Fernandes, A.; Mora, A.; Magrinho, M.; Lopes, A.; Electrochemical degradation of C. I. Acid Orange 7; Dyes Pigments, v.61, p. 287- 296, (2004).

2. Guaratini, C. C. I.; Zanoni, M. V. B.; Corantes Têxteis; Química Nova, Revisão, v.23, n.1, (2000).

3. IUPAC: União Internacional de Química Pura e Aplicada. Reporting Physisoption data for gás/solid systems, v. 54, p. 2201-2218, 1982.

4. Kunz, A.; Zamora, P.P.; Moraes, S.G. E Durán, N.; Novas tendências no tratamento de efluentes têxteis. Química Nova, Vol 25, $\mathrm{N}^{\circ} 1,78$ 82 (2002).

5. Lima, E. C.; Royer, B.; Vaghetti, J. C. P.; Simon, N. M.; Da Cunha, B. M.; Pavan, F. A.; Benvenutti, E. V.; Veses, R. C.; Airoldi, C.; J. Hazard. Mater.; 2008, 155, 536-550.

6. Neta, Maria Rosa de Oliveira. Recursos hídricos: água um bem precioso para a humanidade. Conteudo Juridico, Brasilia-DF: 15 abr. 2013. Disponivel em: <http://www.conteudojuridico.com. br/?artigos\&ver=2.42976\&seo=1>. Acesso em: 28 out. 2017.

7. Regalbuto, Jonh. Catalyst preparation: Science and engineering. 1 ed. New York: CRC Press, 2016. 488p.

8. Rodrigues, L. A.; Silva, M. L. C. P.; Alvares-Mendes, M. O.; Coutinho, A. R.; Thim, G. P. Phenol removal from aqueous solution by activated carbon produced from avocado kernel seeds. Chemical Engineering Journal, v. 174, p.49-57, 2011.

9. Soto, M. L.; Moure, A.; Domingues, H.; Parajó, J. C. Recovery, concentration and purification of phenolic compounds by adsorptionA review. Journal of Foog Engineering, v. 105, n. 1, p. 1 - 27, 2011.

10. Tundisi, José Galizia, Tundisi, Takako Matsumura. Recursos Hídricos no Século XXI. $1^{a}$ ed. São Paulo: Editora Oficina de Textos, 2011.

\section{lara A. Freire*, Gabriel N. C. Nova \& Nelson M. L. Filho}

Universidade Federal de Pernambuco, Departamento de Engenharia Química

*E-mail: iafreirealves@gmail.com 